\title{
Relationship of the characteristics of large Forbush decreases and the heliolongitude of their sources
}

\author{
Maria Papailiou', Maria Abunina ${ }^{(i)}{ }^{2}$, Anatoly Belov ${ }^{(12}$, Eugenia A. Eroshenko ${ }^{+2}$, Victor Yanke ${ }^{\left({ }^{2}\right.}$, \\ Helen Mavromichalaki

\section{Correspondence} \\ 'Nuclear and Particle Physics Department, Faculty of Physics, National and Kapodistrian University of Athens, \\ Greece,mpapahl@phys.uoa.gr, emavromi@phys.uoa.gr \\ ${ }^{2}$ Pushkov Institute of Terrestrial Magnetism, lonosphere and Radiowave Propagation (IZMIRAN), Moscow, \\ Russia, \\ abunina@izmiran.ru,abelov@izmiran.ru,erosh@izmiran.ru,yanke@izmiran.ru
}

\section{OPEN ACCESS}

This work is published under the Creative Commons Attribution 4.0 International licence (CC BY 4.0) Please note that individual, appropriately marked parts of the work may be excluded from the licence mentioned or may be subject to other copyright conditions. If such thirdparty mitens. if such thirdparty materiatis not under the Creative Commons license, any copying, editing or public reproduction is only permitted with the prior consent of the respective copy right owner or on the basis of relevan legal authorization regulations.

\section{Keywords}

Forbush decreases; solar sources; heliolongitude; cosmic ray anisotropy

\begin{abstract}
In this investigation the different features and characteristics of Forbush decreases, with emphasis on large Forbush decreases $(\geq 4 \%)$ and their association to solar sources, are being examined. According to the heliolongitude of the solar source, the events under study were separated into three subcategories: western $\left(21^{\circ} \leq\right.$ heliolongitude $\left.\leq 60^{\circ}\right)$, eastern $\left(-60^{\circ} \leq\right.$ heliolongitude $\left.\leq-21^{\circ}\right)$ and central $\left(-20^{\circ} \leq\right.$ heliolongitude $\left.\leq 20^{\circ}\right)$. The selected events cover the time period 1967 - 2017. The 'Global Survey Method' was used for analyzing the Forbush decreases, along with data on solar flares, solar wind speed, geomagnetic indices (Kp and Dst), and interplanetary magnetic field. In addition, the superimposed epoch method was applied in order to plot the time profiles for the aforementioned group of events. This detailed analysis reveals interesting results concerning the features of cosmic ray decreases in regard to the heliolongitude of the solar sources. Moreover, it is also shown that large Forbush decreases, regardless of the heliolongitude of the solar source, are accompanied by increased geomagnetic activity and increased anisotropy, including anisotropy before the events, which can serve as a typical precursor of Forbush decreases.
\end{abstract}

\section{Introduction}

The Forbush decrease (FD) effect can be defined as 'the result of the effect of coronal mass ejections (CMEs and ICMEs) and/or high speed solar wind streams originating from coronal holes on cosmic rays' (Papaioannou et al. 2010; Belov et al. 2014; Kryakunova et al. 2015). FDs are observed as a short term and steep decrease of the galactic cosmic ray intensity which is followed by a relatively slow recovery (e.g. Melkumyan et al. 2018) lasting up to one week (Cane 2000; Usoskin et al. 2008; Melkumyan et al. 2019). The study of FDs reveals a wide variety of these events in regard to the amplitude and the duration of the events, the anisotropy, the fast or gradual decrease, the complete recovery or absence of recovery phase, the completion in one or two steps, the simple or complicated time profile, etc. The diversity of the solar sources related to them may be one factor that could provide interesting results about the aforementioned variety and the various manifestations of their characteristics. In this study the different features and characteristics of FDs, with emphasis on large FDs and their association to solar sources are being examined. In particular, this 
work focuses mainly on the influence of solar sources position on the FD magnitude (i.e., on the variation of the cosmic ray density) and on the maximum anisotropy of cosmic rays.

\section{Data and method}

The Forbusheffects and interplanetary disturbances database (FEID) of IZMIRAN (http://spaceweather. izmiran.ru/eng/dbs.html, last accessed April 8, 2021) has been used for this study (Abunin et al. 2013). Cosmic ray parameters (density, anisotropy, etc.) in this database are calculated by the 'Global Survey Method' (Belov et al. 2018). In addition to solar-wind parameters (solar wind speed and interplanetary magnetic field intensity) and geomagnetic indices (Ap, Kp and Dst) that were obtained from the OMNI database (http://omniweb.gsfc.nasa.gov, last accessed April 8, 2021), the list of sudden storm commencements (SSCs) (http://isgi.unistra.fr/data_download.php, last accessed April 8, 2021) and of the solar flares data (class, location, time) reported in the solar geophysical data (ftp://ftp.swpc.noaa.gov/pub/indices/events/, last accessed April 8, 2021) were also used (Belov et al. 2014).

\section{Analysis and results}

In this study, the selection of events and their grouping was made using the combination of four criteria. The criterion for group A is the SSC, for group B, apart from the SSC, the magnitude is also considered, whereas the FDs of group C are being investigated with respect to SSC, magnitude, quiet background and presence of an identified solar source. Moreover, the FDs of group C were further divided into three subcategories, according to the heliolongitude of the solar source. As a result, the categories were organized in table 1. In this table, Group C includes 100 events associated with sources with heliolongitude $-90^{\circ}-90^{\circ}$, but since FDs associated with sources from the far eastern $\left(-90^{\circ}--61^{\circ}\right)$ and far western $\left(61^{\circ}-90^{\circ}\right)$ regions are not being considered in the particular study, the remaining 87 FDs are organized in groups $\mathrm{C}-\mathrm{W}, \mathrm{C}-\mathrm{E}$, and C-C.

\begin{tabular}{|l|l|l|}
\hline Categorization & Criteria & Number of events \\
\hline Group A & FDs with SSC & 1515 \\
\hline Group B & FDs with SSC and magnitude greater than 4\% & 335 \\
\hline Group C & $\begin{array}{l}\text { FDs with SSC, magnitude }>4 \% \text {, evolved in a quiet background (no other } \\
\text { events were registered for } 48 \text { hours before and } 18 \text { hours after the onset } \\
\text { of each FD) and connected to identified solar sources: }\end{array}$ & 100 \\
\hline Group C-W & western sources $\left(21^{\circ} \leq\right.$ heliolongitude $\left.\leq 60^{\circ}\right)$ & 23 \\
\hline Group C-E & eastern sources $\left(-60^{\circ} \leq\right.$ heliolongitude $\left.\leq-21^{\circ}\right)$ & 28 \\
\hline Group C-C & central sources $\left(-20^{\circ} \leq\right.$ heliolongitude $\left.\leq 20^{\circ}\right)$ & 36 \\
\hline
\end{tabular}

Table 1: The criteria for grouping the FDs registered from 1967 - 2017 used in this study. 


\subsection{Time profiles of FD groups}

Averaged time profiles for some parameters concerning the aforementioned groups of events were plotted. Specifically, cosmic ray density A0 and the magnitude of the component Axy of the first harmonic of anisotropy along with the interplanetary magnetic field intensity and the solar wind speed and geomagnetic indices Dst and Kp for the groups C-W (figure 1), C-E and C-C were plotted as result of the application of the superimposed epoch method. These profiles cover a time period of 48 hours before and 120 hours after the SSC. In figure 1, the particularity of the changes in density 44 - 30 hours before the onset of the events is not associated with modulation effects but is a consequence of GLEs, which quite often precede large FDs from western solar sources. Four of the selected events are related to GLEs (13 October 1981, 26 October1989, 04 November 2003, 14 December 2006).

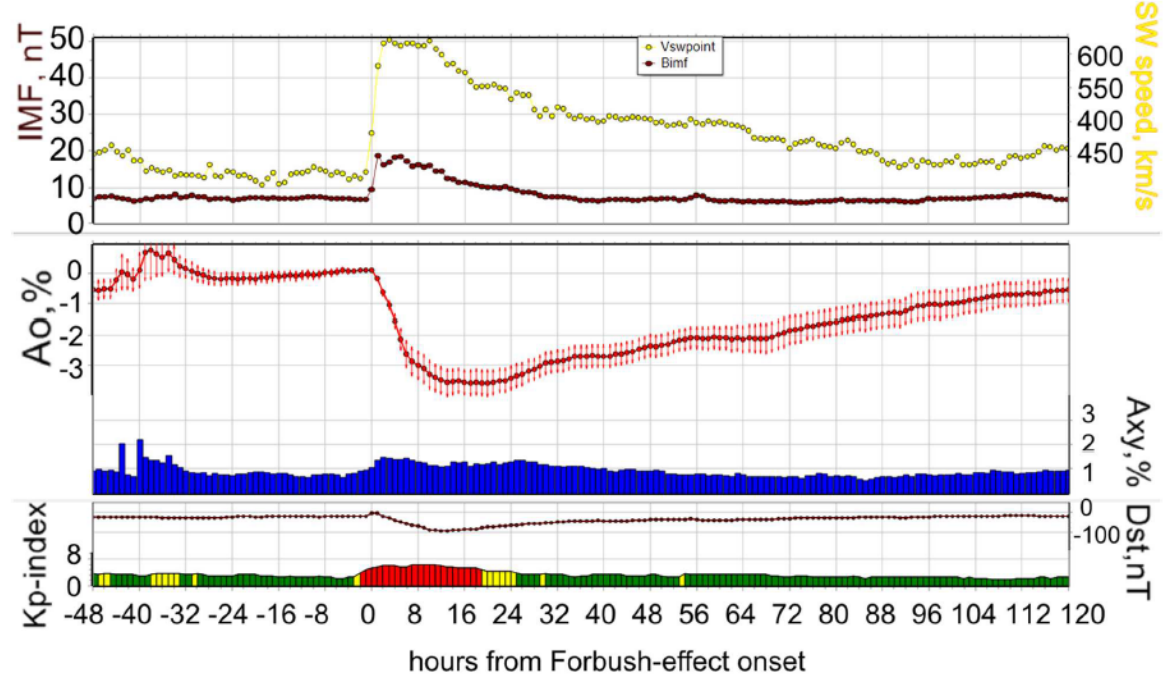

Figure 1: Variations of the interplanetary magnetic field and the solar wind speed (upper panel), the cosmic ray density $\mathrm{A} 0$ (points) and the magnitude of the component Axy of the first harmonic of anisotropy (columns) (middle panel) and the Dst and $\mathrm{Kp}$ indices (bottom panel) for the time periods before and after the arrival of the SSC, as they were calculated for 23 FDs connected to western sources with magnitude $\geq 4 \%$ and SSC by the superimposed epoch method. 0 suggests the recording time of the SSC.

The results from the superimposed epoch method are organized in table 2 . The most interesting parameters are the FD magnitude (AF, \%), the maximum value of the equatorial component of the first harmonic of CR anisotropy $\left(A_{x y m a x}, \%\right)$, the anisotropy one hour before the onset $\left(A_{x y b}, \%\right)$, the minimum value of the geomagnetic index Dst (Dstmin), the maximum value of the interplanetary magnetic field intensity $\left(\mathrm{B}_{\max }\right)$ and the maximum value of the solar wind speed $\left(\mathrm{V}_{\max }\right)$ averaged by the superimposed epoch method over all events and finally, the variation during the last hour before the onset of each event $\Delta \mathrm{B}=\mathrm{B}_{0}-\mathrm{B}_{\mathrm{b}}$ and $\Delta \mathrm{V}=\mathrm{V}_{0}-\mathrm{V}_{\mathrm{b}}$, where $\mathrm{B}_{0}$ and $\mathrm{V}_{0}$ are the interplanetary magnetic field intensity and solar wind speed values at the time of the SSC registration and $B_{b}$ and $\mathrm{V}_{\mathrm{b}}$ are the interplanetary magnetic field intensity and solar wind speed values one hour before the onset of the event respectively. 


\begin{tabular}{|c|c|c|c|c|c|}
\hline \multirow[t]{2}{*}{ Parameter } & \multirow{2}{*}{$\begin{array}{l}1515 \text { FDs } \\
\text { SSC }\end{array}$} & \multirow{2}{*}{$\begin{array}{l}335 \mathrm{FDs} \\
\text { SSC } \\
\text { Magn. } \geq 4 \%\end{array}$} & \multicolumn{3}{|c|}{$\begin{array}{l}87 \mathrm{FDs} \\
\mathrm{SSC}, \mathrm{A}_{\mathrm{F}} \geq 4 \% \text {, quiet background, } \\
\text { identified solar source }\left(-60^{\circ} \leq \text { heliolong. } \leq 60^{\circ}\right)\end{array}$} \\
\hline & & & $\begin{array}{l}\text { Western } \\
\text { sources }\end{array}$ & Eastern sources & Central sources \\
\hline$A_{F} \%$ & $1.75 \pm 0.06$ & $4.57 \pm 0.15$ & $3.7 \pm 0.45$ & $4.37 \pm 0.52$ & $5.01 \pm 0.31$ \\
\hline$A_{x y \max } \%$ & $0.96 \pm 0.02$ & $1.48 \pm 0.05$ & $1.47 \pm 0.19$ & $1.36 \pm 0.18$ & $1.70 \pm 0.15$ \\
\hline$A_{x y b} \%$ & $0.76 \pm 0.01$ & $0.93 \pm 0.04$ & $0.94 \pm 0.14$ & $0.96 \pm 0.09$ & $0.70 \pm 0.06$ \\
\hline Dst $_{\text {min }} n T$ & $-44.2 \pm 1.3$ & $-84.6 \pm 4.17$ & $-92.4 \pm 15.46$ & $-74.6 \pm 12.38$ & $-90.4 \pm 12.1$ \\
\hline $\mathrm{B}_{\max } \mathrm{nT}$ & $13.24 \pm 0.22$ & $19.28 \pm 0.63$ & $18.66 \pm 1.70$ & $20.88 \pm 2.43$ & $20.04 \pm 2.11$ \\
\hline$\Delta \mathrm{BnT}$ & $2.31 \pm 0.20$ & $3.92 \pm 0.48$ & $2.71 \pm 1.10$ & $3.35 \pm 1.17$ & $4.09 \pm 0.97$ \\
\hline $\mathrm{V}_{\max } \mathrm{km} \mathrm{s}^{-1}$ & $492.8 \pm 4.0$ & $603.9 \pm 10.6$ & $619.7 \pm 42.1$ & $583.9 \pm 27.3$ & $627.0 \pm 24.5$ \\
\hline$\Delta \mathrm{V} \mathrm{km} \mathrm{s}^{-1}$ & $25.9 \pm 4.4$ & $42.5 \pm 11.5$ & $56.7 \pm 35.6$ & $25.8 \pm 19.2$ & $59.3 \pm 22.7$ \\
\hline
\end{tabular}

Table 2: Results from the superimposed epoch analysis for the categories of the events under study.

The magnitude of the events connected to western solar sources $(3.7 \%)$ is obviously smaller than that of the events connected to eastern (4.37\%) or central sources (5.01\%) (Abunina et al. 2013a). The typical value for the parameter of anisotropy during quiet conditions is $0.53 \%$ (Abunina et al. 2013b). Moreover, the usual value of anisotropy 1 hour before the FD is 0.7\% (Belov et al. 2008), which agrees with the result for FDs with SSC $(0.76 \%)$ and the FDs related to central sources $(0.70 \%)$. However, for the large FDs there is an observed increase of the $A_{x y b}$ almost up to $1 \%$. As seen in table 2 the anisotropy one hour before the onset of the FD is almost the same (0.94 and 0.96), regardless of the location of the solar source (western or eastern) and its value significantly exceeds the typical value of anisotropy during quiet conditions (Abunina et al. 2013b). This, apparently, is a consequence of an increase of the CR density gradient in this part of the solar wind disturbance. This confirms the assumption that increased vector anisotropy is one of the typical precursors of FDs (Belov et al. 2008; Papailiou et al. 2012).

\subsection{FDs in relation to solar sources}

Average values of different parameters connected to the FDs under investigation are shown in table 3 . Central and eastern sources not only provide more FDs, but also the FDs related to those sources are significantly larger regarding the magnitude in comparison to western sources (Belov 2008).

\begin{tabular}{|c|c|c|c|}
\hline \multirow[b]{2}{*}{ Parameter } & \multicolumn{3}{|c|}{$\begin{array}{l}87 \mathrm{FDs} \\
\mathrm{SSC}, \mathrm{AF} \geq 4 \% \text {, quiet background, } \\
\text { identified solar source }\left(-60^{\circ} \leq \text { heliolong. } \leq 60^{\circ}\right)\end{array}$} \\
\hline & Western sources & Eastern sources & Central sources \\
\hline$A_{F}(\%)$ & $5.16 \pm 0.56$ & $5.59 \pm 0.61$ & $6.64 \pm 0.57$ \\
\hline$A_{x y \max }(\%)$ & $2.58 \pm 0.2$ & $2.22 \pm 0.14$ & $2.73 \pm 0.18$ \\
\hline$A_{x y b}(\%)$ & $1.350 \pm 0.143$ & $1.296 \pm 0.139$ & $1.262 \pm 0.144$ \\
\hline $\mathrm{Kp}_{\max }$ & $6.75 \pm 0.30$ & $6.42 \pm 0.25$ & $6.8 \pm 0.23$ \\
\hline$A p_{\max }(2 n T)$ & $138.6 \pm 15.9$ & $121.6 \pm 15.0$ & $139.9 \pm 13.0$ \\
\hline$D_{s t}{ }_{\text {min }}(n T)$ & $-115.7 \pm 15.3$ & $-105.7 \pm 13.4$ & $-135.9 \pm 14.8$ \\
\hline $\mathrm{B}_{\max }(\mathrm{nT})$ & $22.56 \pm 2.29$ & $24.77 \pm 2.20$ & $27.07 \pm 1.75$ \\
\hline$V_{\text {max }}\left(\mathrm{km} \mathrm{s}^{-1}\right)$ & $660.5 \pm 36.3$ & $614.5 \pm 26.9$ & $660.2 \pm 23.2$ \\
\hline$t_{\min }$ (hour) & $18.35 \pm 2.28$ & $22.61 \pm 2.12$ & $20.42 \pm 1.67$ \\
\hline
\end{tabular}

Table 3: Average values of different parameters of the large FDs related to western, eastern and central sources. 
However, FDs related to western sources are characterized by higher values of $A_{x y b}$ (Papailiou et al. 2013). On the other hand, the maximum anisotropy for central sources is the highest (table 3), but there is a noteworthy difference between $A_{x y m a x}$ for western $(2.58 \%)$ and eastern sources $(2.22 \%)$. FDs connected to central sources evolved in a more disturbed interplanetary medium (highest values for solar wind speed and IMF intensity are $660.2 \mathrm{kms}^{-1}$ and $27.07 \mathrm{nT}$ respectively) and are accompanied by strong geomagnetic activity, i.e. Dstmin $=-135.9 \mathrm{nT}, \mathrm{Kp}_{\max }=6.8$ and $\mathrm{Ap}_{\max }=139.9$. Geomagnetic activity is more pronounced for FDs related to western sources (Dst $t_{\min }=-115.7 \mathrm{nT}, \mathrm{Kp}_{\max }=6.75$ and $A p_{\max }=138.6$ ) rather than those connected to eastern sources (Dst $t_{\min }=-105.7 \mathrm{nT}, \mathrm{Kp}_{\max }=6.42$ and $\left.A p_{\max }=121.6\right)$, although the difference is not so big. Moreover, FDs connected to western sources reach the minimum more rapidly $\left(\mathrm{t}_{\min }=18.35 \mathrm{hrs}\right)$ than those related to eastern or central sources, which develop more slowly ( $t_{\min }=22.61 \mathrm{hrs}$ and $t_{\min }=20.42 \mathrm{hrs}$ ) (Abunina et al. 2013a).

\section{Discussion and conclusion}

This study refers to a group of large FDs, i.e. FDs with magnitude $\geq 4 \%$ for particles of $10 \mathrm{GV}$ which evolve in a quiet background (with a time difference of 48 and 18 hours from the previous and next event respectively), with identified solar sources and which were accompanied by an SSC. The FDs were selected from the IZMIRAN Forbush effects and interplanetary disturbances database and cover the time period from 1967 to 2017. These events were grouped into three categories according to the heliolongitude of their solar sources.

The features of the events with different solar sources are highlighted and summarized as follows: a) Large FDs ( $\geq 4 \%$ ) related to central or eastern sources are more often observed, whereas events from western sources are the rarest, b) The magnitude of the FDs connected to western solar sources seems to be somewhat smaller than that one of eastern or central sources, c) FDs connected to western sources have a shorter life span in comparison to FDs connected to eastern or central sources which present a slower development, d) The averaged anisotropy one hour before the SSC is increased for all groups, e) The anisotropy one hour before the SSC is somewhat greater in FDs related to western sources than those related to eastern or central sources, f) Increased vector anisotropy is one of the typical precursors of FDs, g) FDs related to central sources evolved in more disturbed interplanetary conditions, which is reflected in a larger IMF increase, $h$ ) In general large FDs ( $\geq 4 \%$ ) are accompanied by moderate geomagnetic storms, however, some events are related to severe or even extreme geomagnetic conditions.

\section{References}

Abunin, A., Abunina, M., Belov, A., et al. 2013, J. Physics: Conf. Series, 409, DOI: https://dx.doi.org/10.1088/1742-6596/409/1/012165 Abunina, M., Abunin, A., Belov, A. et al. 2013a, Geomagn. Aeronomy 53, 10, DOI: https://dx.doi.org/10.1134/S0016793213010027 Abunina, M., Abunin, A., Belov, A. et al. 2013b, Geomagn. Aeronomy 53, 561, DOI: https://dx.doi.org/10.1134/S0016793213050022 Belov, A.V., 2008, Gopalswamy, N., Webb, D.F. (eds.), Universal Heliophysical Processes, Proc. IAU Sympos, 257, Cambridge Univ. Press, 439, DOI: https://dx.doi.org/10.1017/S1743921309029676

Belov, A., Dryn, E., Eroshenko, E., et al. 2008, Kiraly, P., Kudela, K., Stehlik, M., Wolfendale, A.W. (eds.), Proc. 21st ECRS, Institute of Experimental Physics, Slovak Academy of Sciences, Kosice, Slovakia, 347

Belov, A., Abunin, A., Abunina, M., et al. 2014, Solar Phys. 289, 3949, DOI: https://dx.doi.org/10.1007/s11207-014-0534-6

Belov, A., Eroshenko, E., Yanke, V., et al. 2018, Solar Phys, 293, 68, DOI: https://dx.doi.org/10.1007/s11207-018-1277-6

Cane, H., 2000, Space Sci. Rev. 93, 55, DOI: https://dx.doi.org/10.1023/A:1026532125747

Kryakunova, O., Belov, A., Abunin, A., et al. 2015, J. Physics: Conf. Series, 632, DOI: https://dx.doi.org/10.1088/1742-6596/632/1/012062

Melkumyan, A., Belov, A., Abunina, M., et al. 2018, Geomagn. Aeronomy 58, 615, DOI: https://doi.org/10.1134/S0016793218050109

Melkumyan, A., Belov, A., Abunina, M., et al. 2019, Solar-Terres. Physics, 5, 28, DOI: https://dx.doi.org/10.12737/stp-51201904

Papailiou, M., Mavromichalaki, H., Belov,A., et al. 2012, Solar Phys. 276, 337, DOI: https://dx.doi.org/10.1007/s11207-011-9888-1

Papailiou, M., Abunina, M., Mavromichalaki, H., et al. 2013, Solar Phys. 283, 557, DOl: https://dx.doi.org/10.1007/s11207-013-0231-x

Papaioannou, A., Malandraki, O., Belov, A., et al. 2010, Solar Phys. 266, 181, DOI: https://dx.doi.org/10.1007/s11207-010-9601-9

Usoskin, I.G., Braun, I., Gladysheva, O. G., et al. 2008, J. Geophys. Res. Space Phys. 113, DOI: https://dx.doi.org/10.1029/2007JA012955 